\title{
CONVEX MAPS
}

\section{S. K. STEIN}

Let $M$ be a planar map, that is, a partition of the plane by simple arcs and curves into a finite number of connected sets, one of which is unbounded, and such that the union of the bounded regions is homeomorphic to a disk. The bounded regions we shall call countries; the union of the bounded regions, the continent. Countries, which we take as closed sets, may touch on arcs or vertices.

We ask the question: when is a map equivalent to a map in which all the countries are convex? More specifically, when can we find a map containing only convex countries, homeomorphic to the countries of $M$ and preserving all incidence relations of $M$ ?

Two necessary conditions come to mind. (a) The countries of $M$ are simply connected. For a convex country is homeomorphic to a disk, hence simply connected. (b) The common border of two touching countries is connected. For the common border of two touching convex countries is a straight line segment, or a point, hence connected. We may combine these two into one statement: no country or union of two countries of $M$ forms a multiply connected set.

Conditions (a) and (b) turn out to be sufficient to make $M$ equivalent to a convex map. Indeed, we shall prove a somewhat stronger theorem.

In order to simplify the statement of the theorem we first dispose of a small difficulty that the shore of the continent may present. The difficulty appears already when $M$ consists of 1 country, 2 edges, and 2 vertices; there are not enough edges and vertices with which to construct a polygon. Therefore we first equip the countries on the shore with sufficient vertices (at least three for each such country). Assumptions (a) and (b) insured that the other countries have at least 3 vertices.

Theorem. $A$ map satisfying (a) and (b) is equivalent to a convex polygonal map in which nonintersecting edges are not parallel.

The key to the inductive proof lies in the following lemma.

LEMмA. If $M$ is a map, has at least two countries, and satisfies (a) and (b), then there are two countries of $M$, say $A$ and $B$, such that $A$ and $B$ touch on an arc and for any country $C$ of $M$ the set $C \cap(A \cup B)$ is connected (possibly empty).

Presented to the Society, June 17, 1950; received by the editors May 23, 1950. 
Proof of the Lemma. Assume the contrary. Then for every two countries $A$ and $B$ that touch on an arc there is a third country $C$ so that $C \cap(A \cup B)$ is not connected. We shall force a contradiction.

Since the continent is homeomorphic to a disk there are two countries, say $A_{1}$ and $A_{2}$, touching on an arc. By our assumption there is a country $C_{1}$ such that $C_{1} \cap\left(A_{1} \cup A_{2}\right)$ is not connected. Since the boundary of $A_{1} \cup A_{2} \cup C_{1}$ consists of two simple closed curves, $J_{1}$ and $J_{2}$, perhaps touching at one or two points, we see that $A_{1} \cup A_{2} \cup C_{1}$ separates the plane into two regions. Assume $J_{1}$ is interior to $J_{2}$. Since the continent is homeomorphic to a disk, there must exist further countries interior to $J_{1}$; at least one of them, say $A_{3}$, touches $A_{2}$ on an arc. Thus there is a country $C_{2}$ such that $C_{2} \cap\left(A_{2} \cup A_{3}\right)$ is not connected. $A_{2} \cup A_{3} \cup C_{2}$ separates the plane into two regions. As above, we get a country $A_{4}$ touching $A_{3}$ on an arc. Proceeding in this way we get an infinite sequence of distinct countries $A_{1}, A_{2}, \cdots$, $A_{n}, \cdots$. But our map has only a finite number of countries. This proves the lemma.

Proof of The Theorem. We shall make an induction on $n$, the number of countries of $M$. For $n=1$ the theorem is immediate. Assume the theorem valid for $n=k-1$. Let $M$ have $k>1$ countries. By the lemma there are two countries $A$ and $B$ touching on an arc, with distinct end points at vertices, say $v_{1}$ and $v_{2}$, and $C \cap(A \cup B)$ is connected for all countries $C$ of $M$.

Make $A$ and $B$ into a single country $D^{\prime}$ by removing the arc between them. This makes $M$ into a map $M^{\prime}$ with $k-1$ countries satisfying (a) and (b). Apply the induction. We now assume that $M^{\prime}$ consists of convex polygonal countries and that distinct edges of $M^{\prime}$ are not parallel.

We wish to introduce $A$ and $B$ by dividing $D^{\prime}$ by a straight line segment. This act will be completely described by locating the vertices $v_{1}^{\prime}$ and $v_{2}^{\prime}$ that are to correspond to $v_{1}$ and $v_{2}$. Depending on the way $v_{1}$ and $v_{2}$ were present in $M$ we have three cases. I. Both $v_{1}$ and $v_{2}$ are of order 3. II. Both $v_{1}$ and $v_{2}$ are of order greater than 3 . III. One of $v_{1}$ and $v_{2}$ is of order 3 , the other of order greater than 3 .

Case I. Since $v_{1}$ and $v_{2}$ are of order 3 we lose all trace of them in removing the arc between $A$ and $B$. Hence we have the freedom to locate $v_{1}^{\prime}$ (and $v_{2}^{\prime}$ ) anywhere along a whole edge (or edges) of $M^{\prime}$ and preserving the incidences of $M$. But the edge $v_{1}^{\prime} v_{2}^{\prime}$ may be parallel to an edge of $M^{\prime}$. To remedy this, displace $v_{1}^{\prime}$ and $v_{2}^{\prime}$ a little, along with the edges incident to them, without disturbing the convexity of any of the countries or introducing any parallel edges. Straight-forward continuity argument shows that this is possible. 
Cáse II. Here incidence permits us no leeway in locating $v_{1}^{\prime}$ and $v_{2}^{\prime}$. However, $v_{1}^{\prime} v_{2}^{\prime}$ may be parallel to an edge. To remedy this, displace $v_{1}^{\prime}$ and $v_{2}^{\prime}$ as in Case $I$.

Case III. Combine methods used in the first two cases.

Finally, since nonintersecting edges of our map are not parallel, the countries we have just introduced to correspond to $A$ and $B$ are not empty sets. The exceptional cases in which $D^{\prime}$ or the country in $M^{\prime}$ adjacent to it has just 2 vertices can be treated separately. This proves the theorem.

REMarks. This proof could easily be extended to show that $M$ could be deformed continuously into a convex map. Also, we could make the continent convex. It is to be noted that the induction gives a practical procedure for making $M$ convex. For maps on a sphere a similar theorem holds.

Our theorem shows that the 3- or 4-coloring problem for general planar maps is equivalent to the coloring problem for convex maps. Now, general maps in 3-space pose no coloring problem; it is easily seen that for every integer $n$ we can produce $n$ countries touching each other nontrivially (braid them). We might wonder whether the coloring problem in 3-space becomes interesting when we restrict the countries to being convex. This question was answered by $\mathrm{H}$. Tietze $[1]^{1}$ and A. Besicovitch [2]. Each produces an example showing that an infinity of colors is still required.

\section{BIBLIOGRAPHY}

1. H. Tietze, Monatshefte für Mathematik und Physik (1905) pp. 211-216.

2. A. Besicovitch, J. London Math. Soc. (1947) pp. 285-287.

Columbia University

1 Numbers in brackets refer to the bibliography at the end of the paper. 